identify likely intervention targets and to inform public health policy.

\section{P96 ETHNIC DENSITY EFFECTS FOR ADULT MENTAL HEALTH: SYSTEMATIC REVIEW AND META-ANALYSIS OF INTERNATIONAL STUDIES}

${ }^{1} \mathrm{~L}$ Becares*, ${ }^{2} \mathrm{M}$ Dewey, ${ }^{2} \mathrm{~J}$ Das-Munshi. 'Social Statistics, University of Manchester, Manchester, UK; ${ }^{2}$ Department of Health Services and Population Research, King's College London, Institute of Psychiatry, Psychology and Neuroscience, London, UK

\subsection{6/jech-2017-SSMAbstracts. 197}

Background Despite increased ethnic diversity in more economically developed countries it is unclear whether residential concentration of ethnic minority people (ethnic density) is detrimental or protective for mental health. This is the first systematic review and meta-analysis covering the international literature to assess ethnic density associations with mental health outcomes.

Methods We systematically searched Medline, PsychInfo, Sociological Abstracts, Web of Science from inception to March 31 st, 2016, for observational studies using search terms for the following study outcomes: Depression, anxiety and the common mental disorders, suicide, suicidality, psychotic experiences and psychosis. We obtained additional data from study authors. We conducted random effects meta-analysis taking into account clustering of estimates within datasets. Metaregression assessed heterogeneity in studies due to ethnicity, country, generation and area-level deprivation. This review is registered with PROSPERO CRD42014012992.

Results Out of 2288 studies identified 41 met inclusion criteria. 12 studies provided data for meta-analysis and metaregression. In the meta-analyses, we found a large reduction in relative odds of psychotic experiences (OR: 0.82 (95\% CI: 0.76-0.89) (nine estimates)) and suicidal ideation (OR: 0.88 (95\% CI: 0.79-0.98) (ten estimates)) for each 10 percentagepoint increase in own ethnic density. For common mental disorders, depression and anxiety, associations were indicative of protective effects of own ethnic density however results were not statistically significant.

Conclusion The findings support consistent protective ethnic density associations across countries and racial/ethnic minority populations as well as mental health outcomes. This is indicative of the importance of the social environment in patterning detrimental mental health outcomes in marginalised and excluded population groups.

\section{P97 THE 'PROBLEM' OF ROMA HEALTH AND WELLBEING: A CRITICAL ANALYSIS OF EUROPEAN POLICY PERSPECTIVES}

LC Orton*, S Sheard. Public Health and Policy, University of Liverpool, Liverpool, UK

10.1136/jech-2017-SSMAbstracts. 198

Background Roma are the largest minority ethnic group in Europe. They have faced ongoing discrimination and persecution, with reports of large and widening social and health disparities between Roma and non-Roma. Most analyses of the policies that aim to improve the situation for Europe's Roma compare the pros and cons of proposed solutions. They do not reveal the assumptions that shape how the "problem" of
Roma health and wellbeing is understood in policy proposals and how this influences the solutions that are - or are notput forward. The aim of this study is to explore the conditions that have allowed certain representations of the "problem" of Roma health-and-wellbeing to acquire dominance/ suppression during the 1980-2015 period of economic transition and successive eastward EU expansion.

Methods Five oral history interviews were conducted with European policy specialists to provide accounts of the development of European policy relevant to Roma health and wellbeing and to map key documents produced by EU decisionmaking institutions (the Parliament, Council and Commission). Further documents were located through catalogue searches. All retrieved items were mapped/categorised before five policies relating to pivotal shifts in the way Roma health and wellbeing has been understood as a policy problem were selected. Analyses drew on Bacchi's "What's the problem represented to be?" post-structural analysis framework to explore: 1) underlying frameworks of thought that shape problem representations; 2) the conditions that allowed shifts in problem representations to occur; and 3) how these problem representations shaped the different solutions that have been proposed.

Results Since the 1980's policies produced by the main EU institutions have situated the concern for Roma health and wellbeing within a wider drive for economic growth. A perceived low productivity among Europe's Roma populations is attributed to their low educational attainment and subsequently reduced employment opportunities. Inadequate housing/living conditions and low uptake of health care services are seen to compound the "problem" leaving Roma prone to poor health that further limits their productivity. The proposed solution is to remove the barriers that are perceived to prevent Roma from accessing mainstream health, education, employment and housing.

Conclusion With a perceived increase in the size and mobility of its Roma populations, recent EU policy has framed "Roma health" as a barrier to economic development. Policy solutions frame improvements in Roma health as a means to improve overall productivity of the region, rather than as an end in itself.

\section{P98 ASSESSING CHANGE IN PHYSICAL ACTIVITY LEVELS IN ADOLESCENT ETHNIC GROUPS}

P Bhatnagar*, N Townsend. Nuffield Department of Population Health, University of Oxford, Oxford, UK

\subsection{6/jech-2017-SSMAbstracts. 199}

Background Previous research has established that physical activity declines during childhood, but we have inadequate information on how these changes differ by ethnic group. Determining if inequalities exist between ethnic groups is vital for understanding whether different intervention strategies are necessary. We aimed to determine how physical activity has changed over time within different UK ethnic groups.

Methods We used Understanding Society, a nationally representative UK-based longitudinal survey to track changes in physical activity over time in different ethnic groups. For children aged 10 to 15 , we explored the frequency of participating in sports and mode of travel to school. These factors were measured in four of the available six waves (1, 24 and 6), 
between 2009 and 2015. Children were asked how frequently they participate in sports per week. For mode of travel, children reported whether they walked, cycled, used public transport, were driven or used a combination of these. When including children who were followed up, the sample size was too low to stratify by age or gender. We used linear regression to assess if ethnicity was associated with change in sports participation and mode of transport to school.

Results At baseline, low sports participation (6\%) and inactive commuting (54\%) was highest in Pakistani children; differences were significant between ethnic groups for mode of travel to school $(\mathrm{p}<0.00)$. Frequency of sports participation declined for all ethnic groups between waves 1 and 2, 1 and 4 and 1 and 6 . The majority of children did not increase or decrease the overall activity level of their mode of transport to school between any of the waves. Most groups continued to use an active method, though between waves 1 and 4, 50\% of Pakistani children retained an inactive method of commuting to school. Despite some differences, linear regression showed that ethnicity was not significantly associated with change in frequency of sports participation or change in mode of transport between any of the waves.

Conclusion Interventions aimed at preventing the decline of physical activity in children from the general population may be appropriate for ethnic minority groups. As active travel to school remains low in some ethnic minorities, targeted interventions to initially change mode of commuting in ethnic minority children is still needed. 